\title{
La construction de l'Europe : le cas des élections européennes finlandaises en 1999
}

\section{Niilo Kauppi}

\section{(2) OpenEdition}

1 Journals

Édition électronique

URL : http://journals.openedition.org/conflits/288

DOI : $10.4000 /$ conflits.288

ISSN : $1777-5345$

Éditeur :

CCLS - Centre d'études sur les conflits lilberté et sécurité, L'Harmattan

Édition imprimée

Date de publication : 1 juin 2000

ISBN : 2-7384-9946-5

ISSN : 1157-996X

\section{Référence électronique}

Niilo Kauppi, «La construction de l'Europe : le cas des élections européennes finlandaises en 1999 », Cultures \& Conflits [En ligne], 38-39 | été-automne 2000, mis en ligne le 20 mars 2006, consulté le 30 mars 2021. URL : http://journals.openedition.org/conflits/288; DOI : https://doi.org/10.4000/conflits. 288

Ce document a été généré automatiquement le 30 mars 2021.

Creative Commons License 


\title{
La construction de l'Europe : le cas des élections européennes finlandaises en 1999
}

\author{
Niilo Kauppi
}

1 Province du royaume suédois pendant près de sept cents ans et grand-duché de l'empire russe pendant cent ans, la Finlande indépendante naît au moment de la guerre civile russe. L'adhésion à l'Union européenne en 1995 en même temps que la Suède et l'Autriche peut être considérée comme l'événement politique le plus important depuis cette indépendance. Quels en ont été les effets politiques? Il n'est pas exagéré de dire que l'adhésion à l'Union européenne a changé profondément le système politique national, les relations entre institutions, citoyens et pouvoir politique, entre politique intérieure et étrangère ${ }^{1}$. Dans le débat public, ces effets ont été très peu discutés; ceci ne semble pas un cas isolé dans l'Union Européenne. Parler ouvertement d'Europe semble hors sujet. Le croque-mitaine du fédéralisme lève la tête. Cependant, une européanisation des champs politiques nationaux est bien en route. Structurellement, il s'agit de la constitution d'un secteur commun, hybride de supranational, national et régional, aux quinze champs nationaux. Cette européanisation a deux caractéristiques principales: d'une part une monopolisation supranationale et nationale du pouvoir politique par les instances européennes et l'exécutif politique national et une nouvelle alliance stratégique entre les deux, d'autre part la constitution de nouvelles institutions et de nouveaux réseaux entre niveaux local, régional, national et supranational. Ces processus ont été accompagnés par des constructions symboliques collectives de l'Europe. Pour saisir les effets concrets et la signification politique de l'intégration européenne sur les champs politiques nationaux il faut se déplacer du niveau macro à celui des pratiques sociales des agents politiques. Pourquoi la Finlande ? C'est un cas intéressant car il nous permet d'examiner sur une période de temps limitée, de 1995 à 2000, les effets de l'intégration européenne. Ces effets ne sont évidemment ni toujours clairs ni univoques. Certains observateurs ont parlé d'une intégration accélérée, d'une sur-européanisation induite par une extériorité historique au processus d'intégration européenne ${ }^{2}$. Les cas de la Finlande et de la Suède ont, de cette manière, été distingués 
des premiers Etats membres de la Communauté européenne comme la France, impliqués dans le processus d'intégration depuis le début. Cette intégration accélérée est visible surtout dans les domaines réglementaires et commerciaux, particulièrement en ce qui concerne les privatisations d'entreprises et le triomphe des principes économiques néolibéraux. Cependant, peut-on supposer que les effets de l'intégration européenne sont tangibles au moment où le pays en question entre dans l'Union? Plusieurs changements, comme ce fut le cas de la politique macroéconomique à cause des critères d'adhésion, ont eu lieu avant 1995 pour anticiper et se préparer aux réalités de l'intégration à partir de $1995^{3}$. L'européanisation est un processus qui englobe le champ politique finlandais dans sa totalité, et influence directement et indirectement des changements structurels et discursifs multiples qui ne se limitent pas au système électoral. Cette influence varie de la détermination directe à un support latent dû par exemple à des affinités structurelles entre agents politiques comme, par exemple, les europarlementaires. A titre d'exemple, selon la hiérarchie des ambassades au ministère des Affaires étrangères finlandais, les postes importants ont traditionnellement été Moscou, Stockholm et Washington. Avec l'adhésion à l'Union européenne, Bruxelles doit être ajoutée à cette liste. C'est même, selon certains diplomates, désormais le poste le plus important ${ }^{4}$. L'européanisation du champ politique finlandais signifie que les agents politiques créent diverses stratégies d'européanisation, des stratégies qui reflètent différentes manières de s'approprier l'Europe et les changements que l'intégration impose. Il s'agit à la fois de réactions et d'anticipations locales, de calculs liés aux carrières politiques, de discours politiques et par exemple de l'adoption d'un nouveau vocabulaire politique européen et non plus simplement national. De manière générale, «l'Europe » fonctionne pour beaucoup comme un multiplicateur de pouvoir politique, surtout au niveau de l'exécutif politique national dont les membres peuvent désormais se présenter non seulement comme hommes d'Etat mais bien plus comme hommes d'Etat européens, relativement indépendants du niveau national et insérés dans des réseaux européens et globaux. Dans un monde devenu de plus en plus petit, la valeur de ce capital politique européen s'accroît par rapport au capital politique traditionnel, sans toutefois simplement se substituer à lui ${ }^{5}$. L'exemple de la trajectoire politique du commissaire finlandais Erkki Liikanen l'illustre. Homme politique socialiste, ancien ministre, nommé au poste de commissaire européen en 1995 et renommé en 1999, il est considéré aujourd'hui comme le principal présidentiable à gauche: spectaculaire métamorphose en cinq ans seulement. Les élections au Parlement européen jouent un rôle tout à fait central dans la construction collective de l'Europe et des institutions communautaires. Elles permettent aux organisations politiques, surtout aux partis politiques mais aussi aux mouvements sociaux comme nous le montrent les dernières élections européennes françaises, de construire leur Europe et, ce faisant, d'influencer les perceptions populaires concernant le continent. Les élections européennes contribuent aussi à la restructuration des champs politiques nationaux, en présentant aux agents politiques une nouvelle base d'action politique. Comment conceptualiser le rôle des élections européennes dans ces champs? Les politologues allemands Reif et Schmitt ont présenté le modèle le plus influent du rôle des élections européennes ${ }^{6}$. Selon eux, les élections au Parlement européen sont des élections de second ordre comparées aux élections nationales. Les élections européennes ont les caractéristiques suivantes: 1) Les enjeux politiques sont plus modestes dans ces élections (less at stake) que dans d'autres élections nationales. 2) Les élections de second ordre sont des élections de protestation ce qui explique le taux 
élevé d'abstentions. 3) Les petits et les nouveaux partis ont plus de succès dans les élections de second ordre que dans les élections de premier ordre. 4) Les élections de second ordre signifient souvent une baisse de popularité des partis au pouvoir. Tout en examinant brièvement les campagnes et les élections finlandaises de 1999 nous allons aussi «tester " les idées présentées par Reif et Schmitt. Les règles du jeu Le premier contingent d'europarlementaires finlandais a été choisi en 1995 directement par le Parlement national lors de l'adhésion du pays à l'Union. En 1996, le suffrage universel est appliqué aux élections européennes en Finlande. L'élection de ces seize parlementaires européens s'effectue dans une circonscription unique au scrutin proportionnel de liste avec vote préférentiel. Ceci favorise les petits partis au détriment des grandes formations politiques. Chaque parti ou liste commune peut présenter au plus vingt candidats. Par contre, les élections au Parlement national (eduskunta) se déroulent dans quinze circonscriptions, avec scrutin direct et uninominal. Aux européennes, les électeurs votent pour des individus et non pour des listes. En France, par contre, les élections au Parlement européen sont essentiellement cadrées comme une continuation de la politique des partis politiques ${ }^{7}$. En raison du mode d'élection français, les partis ou les listes communes décident qui seront les candidats sérieux et donc qui sera de facto éligible. Les élections finlandaises ne sont donc plus une continuation de la politique des partis comme en France mais acquièrent au contraire une logique nouvelle propre. Par conséquent, en raison du découpage électoral et du vote préférentiel, le pouvoir des partis dans la sélection des candidats et des candidats éligibles diminue au profit du pouvoir des médias, surtout des trois chaines de télévision nationales et du plus grand quotidien Helsingin sanomat ${ }^{8}$. D'autre biais sont introduits dans le jeu politique par ce système de vote préférentiel. Les organisations politiques collectives, partis et listes, s'engagent dans une course pour le recrutement des candidats jouissant d'une forte visibilité nationale. Par conséquent, les candidats éligibles et éventuellement élus sont généralement des personnes connues du grand public. Certaines peuvent même avoir une expérience politique négligeable ou inexistante. Cette présélection donne un ton nouveau à la compétition politique. Premièrement, le capital économique des candidats et des élus renforce la distance entre élus et citoyens ordinaires. En 1999, en moyenne, les revenus imposables annuels en Finlande des seize élus au Parlement européen sont, comme ceux élus au Parlement national, plus de trois fois supérieurs au revenu moyen des électeurs (environ 52.700e/ 15.600e) ${ }^{9}$. Il est intéressant de noter que parmi les cent quarante candidats aux élections, le revenu imposable moyen est de 34.500e, donc deux fois les revenus des électeurs. Deuxièmement, le rôle du capital scolaire est encore plus crucial dans l'élection des europarlementaires que dans celui des députés nationaux. 13.9\% des électeurs ont un diplôme supérieur alors que $87.5 \%$ des europarlementaires sont passés par l'université. Le taux de détenteurs d'un diplôme supérieur parmi les candidats est de $65 \%$. Seulement $70 \%$ des parlementaires nationaux ont un diplôme supérieur. Troisièmement, les médias, surtout la télévision en tant que présélecteur des candidats, favorisent ceux ayant une bonne présentation de soi. Ces ressources de présentation de soi sont évidemment inégalement distribuées dans la classe politique. Les électeurs choisissent leurs élus sur la base d'une présélection qui met en valeur trois types de capitaux : le capital économique, scolaire et de visibilité. Le taux très élevé de diplômés du supérieur et de célébrités nationales ou régionales parmi les candidats et les élus démontre que les critères de représentation européenne diffèrent de ceux de la représentation politique traditionnelle qui régissent les élections parlementaires et 
municipales, contrôlées par les partis politiques. Comparées aux autres élections, les élections au Parlement européen ressemblent, de par leur caractère d'élections d'individus, aux élections présidentielles. Dans certains pays de l'Union européenne, comme en France, certains hommes politiques comme Michel Rocard ont essayé d'utiliser les élections européennes comme une primaire présidentielle. En Finlande, plusieurs facteurs socioculturels soulignent le caractère individuel des élections européennes. Depuis déjà longtemps en Finlande, les électeurs sont devenus de plus en plus mobiles et la fidélité à un parti est une affaire du passé ${ }^{10}$. Par conséquent, au lieu de garder jalousement leur électeurs, les partis essayent d'attirer de nouveaux électeurs en recrutant dans leurs listes des candidats renommés. Comme dans les autres petits pays de l'Union européenne, le nombre limité de candidats (140) et d'élus (16) finlandais aux élections européennes favorise l'individualisation de l'élection. Le vote préférentiel, en canalisant les voix critiques à l'intérieur des partis, et la stratégie de l'exécutif politique de minimiser la portée des élections et de ce fait, la légitimité du Parlement européen dans la construction européenne, peuvent empêcher la constitution d'un parti ou d'un mouvement anti-européen protestataire fort qui diviserait l'espace des partis politiques et l'électorat. Du coup, la fragmentation des opinions à l'intérieur des partis empêche, au contraire, la constitution d'une ligne de parti unique. Le vote préférentiel a de nombreux effets. Il accentue à l'intérieur des partis la division entre anti- et pro-européens en créant des duels politiques entre candidats "souverainistes" et "fédéralistes". Il aggrave la division entre professionnels du politique, les représentants des partis, et amateurs, "honnêtes gens » représentant la Finlande « là-bas en Europe ». A cause des règles du jeu pour les européennes, les médias deviennent une arène de compétition moins entre les programmes politiques des partis qu'entre les célébrités recrutées par les partis mais se déclarant «indépendantes». Le vote préférentiel souligne aussi la division géographique entre la région capitale d'Helsinki (où habite un cinquième de la population finlandaise) et le reste du pays. Pour cette raison, dans certaines régions, plusieurs groupes d'intérêts se mobilisent pour soutenir des candidats locaux qui peuvent représenter à Bruxelles les intérêts de la région. Indirectement, le rôle des médias, surtout de la télévision, augmente les budgets des candidats, ce qui distingue nettement les élections européennes des élections au Parlement national. Les budgets moyens aux élections parlementaires nationales se situent en 1996 de 25.000 è 34.000 e. Pour les européennes en 1999 la moyenne s'élève à 67.000e, donc plus du double de la moyenne des élections nationales. Le budget du candidat régional centriste Kyösti Virrankoski est astronomique selon les critères locaux, 150.000e. Le rôle central joué par les médias, et surtout la télévision, explique en partie cette escalade des coûts de campagne ${ }^{11}$. Depuis les premières élections au Parlement européen, les programmes des partis se sont considérablement développés. En 1996, tous souffrent d'un manque de vision en ce qui concerne la politique européenne finlandaise. Étant donné le caractère récent de l'adhésion, ceci n'est pas surprenant. Les thèmes des campagnes sont donc neutres ${ }^{12}$. Ceci peut être partiellement expliqué par le sentiment populaire anti-européen, ce qui signifie que les partis laissent aux candidats le travail symbolique de formulation de leur vision européenne. Pour les élections de 1999, les programmes sont déjà nettement plus développés. A droite, les représentants du parti conservateur et du parti centriste conceptualisent l'Europe en terme de projet national, de protection de l'emploi, de solution au problème de la pollution. Surtout parmi les conservateurs les aspects économiques sont populaires : l'Europe garantit la croissance 
économique et stabilise la position économique de la Finlande dans le monde. Le parti conservateur veut rationaliser le système de décision européen sans attribuer plus de pouvoir aux instances communautaires. Le parti centriste, qui est l'ancien parti agraire, s'oppose à la fédéralisation de l'Europe tout en défendant les intérêts des régions. Les centristes, partisans d'une Finlande neutre, veulent la décentralisation du pouvoir sur le continent européen. Le parti suédophone, troisième parti de droite, partage avec les partis de gauche, les sociaux-démocrates et l'Alliance de la gauche (l'ancien Parti communiste), un souci pour la sécurité européenne et la création d'un projet de paix. Cependant, contrairement aux partis de gauche, le parti de la minorité suédophone souligne le rôle positif de la société de marché dans ce projet de paix et de prospérité. Le programme des écologistes s'est précisé depuis 1996. L'Europe a besoin d'un pouvoir supranational pour s'occuper de la protection de l'environnement. Les verts exigent de l'Europe une stratégie commune pour abandonner l'énergie nucléaire. Dans le programme de l'Alliance de la gauche retentit l'Internationale. Moins idéologues, les sociaux-démocrates sont les défenseurs du principe d'égalité dans les décisions européennes et de l'élimination du chômage. En 1999, les programmes des partis se ressemblent. Les différences sont visibles plus au niveau des candidats individuels que des partis politiques. Les plus pro-européens sont les conservateurs, les plus antieuropéens les centristes, en opposition gouvernementale. Entre les deux, les européens critiques, les verts et les suédophones. En raison du mode d'élection, les politiques européennes se forment autant à l'intérieur des partis entre candidats de différentes tendances qu'entre les partis politiques. Le plus grand parti était le "parti Finlande " car, comme en 1996, en 1999 la grande majorité des candidats était choisie pour représenter le pays et non une idéologie politique.

2 Le débat médiatique Sur la chaîne principale de la télévision finlandaise TV1 le " grand débat final » sur les élections a lieu le 10 juin 1999. Les dirigeants politiques de premier rang, premier ministre et chef du parti social-démocrate Paavo Lipponen ainsi que le ministre des finances et chef du parti conservateur Sauli Niinistö, brillent par leur absence. Les partis politiques semblent vouloir éviter de parler de l'Union européenne pendant toute la campagne. Pourquoi ? Le silence peut s'expliquer par le consensus au sein de l'exécutif politique national sur la politique européenne finlandaise. L'anticipation de la présidence finlandaise de l'Union européenne qui devait commencer début juillet, deux semaines après les élections au Parlement européen, fournit une autre raison pour ce silence. Depuis le début de la campagne, le mépris total envers les élections de la part des dirigeants politiques de premier rang est flagrant. Le message aux électeurs est clair : les élections au Parlement européen ne sont que des élections de second ordre; dans l'Union européenne, c'est la coopération intergouvernementale qui compte, surtout pour un petit pays comme la Finlande; le Parlement ne joue qu'un rôle secondaire. Il est vrai que les seize sièges finlandais ne représentent qu'une insignifiante portion du total de 626 sièges du Parlement européen. Le commentaire de Paavo Lipponen lors des premiers résultats des élections est révélateur : « Häkkinen a gagné ! ». Lipponen faisait allusion à la victoire en formule 1 de Mika Häkkinen, un résultat qui semblait plus important pour lui que celui des élections. Le mépris à peine caché de quelques dirigeants politiques envers le processus démocratique est accompagné par l'absence des partis politiques dans le cadrage et l'initiation des débats pendant la campagne. Au lieu de remplir leur rôle politique, les partis ont simplement laissé la formulation des débats et des enjeux aux candidats et aux médias. Par conséquent, les entreprises politiques collectives finlandaises n'ont pas 
élaboré de vision de l'Europe, mais ont de facto laissé ce travail symbolique aux candidats. Cette désertion des partis politiques empêche le développement d'un débat public et politique au sens traditionnel du terme sur l'Europe. Paradoxalement, grâce à cette délégation, le débat médiatique lors de la campagne est centré sur des questions européennes et non de politique intérieure comme c'est le cas pour les élections européennes dans la plupart des pays de l'Union européenne. En effet, ce retrait des partis politiques et des dirigeants politiques de premier rang laisse la porte ouverte à de véritables discussions sur des sujets comme l'élargissement à l'est, le système monétaire européen, le développement des systèmes de décision supranationaux, le rôle du Parlement européen ainsi que du Conseil des ministres. Mais ces débats ont lieu à l'initiative des candidats et des médias sans caution des partis politiques ${ }^{13}$. Les résultats Le 13 juin, le taux de participation est après la Grande Bretagne le plus bas en Europe, seulement $31,4 \%$. Comment expliquer cet effondrement d'un taux de $60,3 \%$ en 1996 à 31,4\% trois ans plus tard ? La non-présence des dirigeants politiques dans la campagne signale aux électeurs que ces élections n'ont que peu d'importance. Le manque d'enjeu politique clair renforce cette perception populaire. De plus, dans la culture politique finlandaise, les affaires européennes sont traditionnellement assimilées à la politique étrangère, domaine réservé des élites et non du peuple. La fatigue électorale est de même visible, les élections au Parlement national ayant eu lieu en mars 1999 avec la participation de 68,3\% des électeurs. Selon une étude du Centre statistique de Finlande ${ }^{14}$, aux européennes les abstentionnistes se trouvent plutôt parmi les électeurs des classes modestes. Cette abstention se reflète directement dans les résultats électoraux des partis de gauche, les sociaux-démocrates et les excommunistes, qui perdent des sièges. Des électeurs de moins de 26 ans, seuls $26 \%$ ont voté. Le taux de participation le plus élevé est dans la région capitale, $40,5 \%$, et les électeurs les plus flegmatiques se trouvent dans la région de Kuopio à l'est du pays $(25,7 \%)$.

3 Les seize élus au Parlement européen sont les suivants: Tableau 1. Les europarlementaires finlandais 1999-2004 (gauche-droite, européanistes-antieuropéens ; les hésitants entre parenthèses)

\section{GAUCHE DROITE}

5 EUROPEANISTES Riitta Myller (SDP, PSE) Ulpu Iivari (SDP, PSE) Heidi Hautala (Verts, V) Reino Paasilinna (SDP, PSE) Piia-Noora Kauppi (Coalition, PPE) Ari Vatanen (Coalition, PPE) Marjo Matikainen-Kallström (Coalition, PPE) Ilkka Suominen (Coalition, PPE) Astrid Thors (parti suédophone, ELDR)

6 ANTI-EUROPEENS (Matti Wuori, Verts, V) Esko Seppänen (Gauche, GVE-NGL) (Eija-Riitta Korhola, Chrétiens, PPE) Mikko Pesälä (Centre, ELDR) Samuli Pohjamo (Centre, ELDR) Kyösti Virrankoski (Centre, ELDR) Paavo Väyrynen (Centre, ELDR)

7 Le prototype de l'europarlementaire est un homme de 47 ans, de la région capitale, détenteur d'un diplôme supérieur, dont les revenus imposables annuels sont d'environ 52.000 e/an. Il fait partie de l'élite sociale aussi bien d'un point de vue économique que scolaire. Le diplomate populaire est un nom connu par le grand public qui a un capital international (linguistique et culturel) important. Trois des seize élus sont des amateurs politiques complets, l'ancien champion de rallye Ari Vatanen, résident de Monaco, recruté par le parti conservateur (Kokoomus, KOK), la journaliste de télévision Eija-Riitta Korhola, élue des rangs du parti chrétien (Suomen kristillinen liitto, SKL) et Matti Wuori, représentant des Verts (Vihreät, VIHR), ancien directeur de Greenpeace 
international et célèbre défenseur des Droits de l'Homme. Tout les trois se présentent comme des patriotes prêts à se battre pour les intérêts du pays au Parlement européen. Wuori déclare se consacrer aux questions d'environnement et aux Droits de l'Homme. Vatanen se présente comme un homme du peuple mais aussi comme un cosmopolite. Dans une interview à Libération, il déclare : «Je ne suis pas un politique. Les gens de la rue ont confiance en moi et m'écoutent quand je leur parle de l'Europe que je connais. J'ai eu le coup de foudre pour la Provence. Nous vivons sur un continent d'une richesse absolument incomparable. Je souhaite construire des ponts entre les gens, être une sorte d'ambassadeur" ${ }^{15}$. Aux élections précédentes en 1996, quatre amateurs politiques sont élus parmi lesquels l'ancienne championne du monde de ski de fond Marjo Matikainen-Kallström (conservateur), réélue en 1999. Parmi les europarlementaires de 1999 figurent, comme en 1996, trois anciens ministres et neuf anciens députés nationaux ( $56 \%$ du contingent), indiquant qu'il existe un lien solide de recrutement direct du parlement national au Parlement européen. 39,3\% (55/140) des candidats et 44\% (7/16) des élus sont des femmes, une bonne moyenne en Finlande. 37\% des élus sont des représentants régionaux, 63\% nationaux. Par exemple, Ari Vatanen, candidat du parti conservateur, reçoit la plupart de ses voix du sud de la Finlande et seulement 1000 voix du nord de la Finlande, de la Laponie. Par contre, 67,7\% des voix du candidat du centriste Kyösti Virrankoski viennent de la région de Vaasa à l'ouest du pays. Parmi les europarlementaires, les élus régionaux sont les centristes Paavo Väyrynen, avec les voix de la Laponie, Kyösti Virrankoski, représentant de l'Ostrobothnie, Samuli Pohjamo de la région de Oulu au nord de la Finlande, Mikko Pesälä de la région de Kymi à l'est du pays, et la social-démocrate Riitta Myller, représentante de la Carélie du Nord à l'est du pays. Les quatre premiers, choisis sur les listes du parti centriste dans l'opposition, sont des anti-européens convaincus, surtout Väyrynen, ancien premier ministre et ancien leader du parti centriste. Myller, par contre, se profile pendant sa campagne comme une social-démocrate pro-européenne, travaillant pour l'intérêt non seulement du parti, mais aussi de la région et du pays. Les 12 autres europarlementaires sont élus grâce aux voix du sud, la partie la plus peuplée du pays. Ceux qui reçoivent le plus de voix sont l'europarlementaire verte Heidi Hautala, avec $9,3 \%$ de tous les votes, l'europarlementaire conservatrice Marjo Matikainen-Kallström, avec 8,6\% des voix et l'europarlementaire du parti suédophone Astrid Thors, avec $6,5 \%$ de tous les votes. Ce palmarès indique la continuité qui existe entre les europarlementaires sortants et les nouveaux élus et la domination des candidats du sud du pays. Hautala est particulièrement populaire dans les grandes villes du sud, Helsinki, Tampere et Turku, les régions où il y a le moins d'abstentions et où pratiquement toutes les élections nationales se jouent. En 1999, le manque de sentiments anti-européens forts empêche les critiques populistes de l'Union européenne, le centriste Paavo Väyrynen et l'ex-communiste Esko Seppänen, de renouveler leur victoire électorale écrasante de 1996. En pourcentage de votes, les élections au Parlement européen démontrent quelques tendances de plus longue durée dans le cycle des élections finlandaises. Tableau 2. Les résultats en pourcentages des votes et de sièges (entre parenthèses). SOC. CENTRE CONSER. GAUCHE SUEDOIS VERTS CHRETIENS PN 1995 28,3 19,8 17,9 11,2 5,1 6,5 - PE 1996 21,5 (4) 24,4 (4) 20,2 (4) 10,5 (2) 5,7 (1) 7,6 (1) 2,8 (-)

PN 1999 22,9 22,4 21 10,9 5,1 7,3 2

9 PE 1999 17,8 (3) 21,3 (4) 25,3 (4) 9,1 (1) 6,8 (1) 13,4 (2) 2,4 (1) 


\section{PN : Parlement national PE : Parlement européen}

Ce tableau indique que dans la popularité des partis politiques depuis 1995 les grands partis comme les sociaux-démocrates et le parti centriste ont perdu tandis que le parti conservateur a gagné du terrain. Comparée aux élections européennes précédentes, la popularité du parti conservateur a augmenté de 5,1\%. Le quatrième parti du pays, qui prend souvent part à la majorité gouvernementale, ne semble plus être l'Alliance de la gauche mais plutôt les verts, qui ont, par rapport aux élections européennes précédentes, gagné 5,8\%. Grâce à leur candidat très visible dans les médias, Astrid Thors, le parti suèdophone réussit à améliorer son score au dessus de sa représentation dans la population finlandaise ( $6 \%$ de la population est suédophone). En termes de sièges, les perdants sont les sociaux-démocrates (de quatre à trois sièges) et l'Alliance de la gauche (ex-communistes) (de deux à un siège). Les gagnants sont, en revanche, les verts (deux au lieu d'un siège) et l'Union chrétienne finlandaise (un siège). Tous les élus des partis gagnants sont des figures nationales connues par le public. Ces données ne semblent pas confirmer les thèses de Reif et de Schmitt concernant le succès dans les élections européennes de l'opposition gouvernementale, dans ce cas le parti centriste, ni le succès des partis protestataires, dans le cas finlandais tout à fait négligeable. Un modèle structurel des transformations des champs politiques nationaux dans le contexte de l'Union européenne en tant que nouveau système politique devrait prendre en compte la position structurelle et temporelle des élections dans le champ et cycle électoral. Il faudrait rapprocher les caractéristiques des hommes politiques travaillant dans les institutions européennes, institutions marginales dans les champs politiques européanisés, et les hiérarchies politiques nationales, pour évaluer les effets de l'intégration européenne sur les champs politiques nationaux. Peut-on parler de la constitution en Finlande d'une élite politique européenne? Il semble en effet qu'une monopolisation par certains agents politiques des postes d'europarlementaires a eu lieu. Les élus suivants réussissent à renouveler leurs mandats en 1999: chez les centristes Paavo Väyrynen, Samuli Pohjamo, Kyösti Virrankoski, au parti socialdémocrate Riitta Myller et Reino Paasilinna, chez les conservateurs Marjo MatikainenKallström, dans l'Alliance de la gauche Esko Seppänen, parmi les verts Heidi Hautala et dans le parti suédophone Astrid Thors. Le taux de renouveau des europarlementaires finlandais est de 56\% (9/16). Qu'en est-il de l'usage politique du Parlement européen? Pour plusieurs d'entre eux, particulièrement Väyrynen et Seppänen, le Parlement européen fonctionne comme une base d'attaque contre les leaders de leurs partis respectifs. Tous les deux sont de virulents critiques de l'Union européenne et du «pragmatisme pro-européen» de l'exécutif politique finlandais. Pour les hommes politiques centristes, le Parlement européen est l'endroit où ils peuvent défendre les intérêts de leur région face à l'Etat national. Les autres parlementaires renouvelant leur mandat sont des pro-européens utilisant leur compétence politique pour construire de nouvelles carrières politiques. Ils unissent niveaux national et transnational en se spécialisant dans des sujets européens. Par exemple, Heidi Hautala a fait sa marque au Parlement européen en tant que spécialiste des questions de protection de l'environnement. Un nouveau type politique : le diplomate populaire Selon un sondage effectué par le plus grand quotidien finlandais Helsingin sanomat juste avant les élections européennes, $83 \%$ des répondants considèrent que le travail le plus important pour un europarlementaire est de poursuivre consciemment les intérêts des Finlandais ${ }^{16}$. Selon ce même sondage, une majorité des personnes interrogées pense que l'europarlementaire doit pouvoir bien s'exprimer et avoir une expérience 
internationale. Avec les élections européennes un nouveau type politique est né dans le champ politique finlandais : le diplomate populaire, hybride d'ambassadeur culturel et de représentant politique. Ce diplomate populaire est élu pour représenter le pays ou une région, pas une idéologie politique, au Parlement européen et pour défendre l'intérêt des Finlandais. Dans le champ politique finlandais, les critères de la représentativité européenne sont culturels au sens large du terme. La personne en question doit être présentable «là-bas » en Europe, être de préférence photogénique, cultivée et polie. En politique comme dans d'autres secteurs de la société finlandaise contemporaine, l'international semble être le monopole des classes moyennes et supérieures. Le cas de Eija-Riitta Korhola illustre la signification des qualités "personnelles». Journaliste de télévision populaire, mère de trois enfants, elle a un DEA et n'a jamais été candidate politique. Elle ne se prend pas pour une politique. «Je me considère plutôt comme une citoyenne critique ${ }^{17}$. Selon elle, le parti chrétien cherchait un(e) candidat(e) extérieur(e) à la politique qui pouvait attirer les voix des électeurs chrétiens. Ce qu'elle fit admirablement. Le quotidien Iltalehti l'a décrite de la manière suivante : «Eija-Riitta Korhola est une représentante idéale pour les chrétiens. Elle a la parole facile, sourit presque tout le temps à l'américaine, elle est élégante et sûre d'elle " ${ }^{18}$. Le cas du candidat centriste régional de la région d'Ostrobothnie à l'ouest du pays Kyösti Virrankoski est aussi exemplaire à plusieurs égards. La campagne de Virrankoski est très fortement soutenue par le plus grand quotidien régional Ilkka. Son élection est présentée comme une nécessité pour l'avenir de la région. Le journal local Ilkka a brossé le profil électoral de Virrankoski : «C'est un prof de maths avec un diplôme supérieur. Kyösti n'est pas un professionnel de la politique. Au contraire il a fait sa carrière comme prof de maths. Mais il s'est occupé pendant huit ans des affaires publiques, d'abord comme aide à Veikko Pihlajamäki au ministère de la défense, puis pendant quatre ans en tant que député. Il connaît les règles du jeu politique, comment on s'occupe des affaires publiques et comment on les influence... Virrankoski sait comment opérer dans l'Union européenne. Il a étudié la machinerie à Bruxelles pendant un mois et la façon dont les initiatives de la région font leur chemin dans la superbureaucratie... il sait ouvrir des portes qui profitent à la région. Un amateur ne doit pas aller au Parlement. Il faut connaître au moins une langue étrangère couramment - l'anglais est de loin la plus importante. Köpi (surnom de Kyösti, NK) parle l'anglais sans problèmes. Dernier point qui n'est pas à négliger même si on en parle peu actuellement: Kyösti est absolument un homme honnête... Si quelqu'un doute que dans ce pays de buveurs de vin un homme simple de principe puisse se débrouiller, et bien le doute est inutile. Au contraire, l'administration de nos affaires communes en Europe ne serait être dans de meilleures mains quand notre représentant se lève le matin avec la tête claire. C'est vrai que Köpi n'est ni un charmeur ni un orateur charismatique. Mais c'est un représentant sur qui on peut compter. C'est ce dont la région a besoin pour ce boulot » ${ }^{19}$. Diplômé du supérieur et enseignant parlant couramment l'anglais, Kyösti Virrankoski est donc un homme politique sans être un professionel du politique, un honnête homme sur lequel on peut compter, quelqu'un qui sait comment les choses marchent à Bruxelles. Les qualités personnelles de Virrankoski prennent le devant par rapport aux qualités politiques du représentant du parti centriste, critère tout à fait secondaire et même a priori néfaste dans ces élections. Virrankoski représente donc toute la population dans cette région. Cette représentation non-politique au sens traditionnel du terme s'est développée à cause du retrait des partis politiques et de la stratégie de délégitimation des élections 
européennes de la part de l'exécutif politique national, les ministres et les dirigeants des partis. Dans les élections européennes finlandaises de 1999, la stratégie de l'exécutif politique national est claire: délégitimer les élections au Parlement européen en laissant les débats et la campagne aux candidats et aux médias. De cette façon, le monopole politique de l'exécutif national est sauvegardé. Les effets de ce retrait sont en partie paradoxaux : pendant la campagne, les débats étaient de fond et portaient sur des sujets européens sans être une continuation des débats politiques intérieurs comme c'est le cas dans la plupart des pays de l'Union européenne. La construction sociale de l'Europe dans les campagnes européennes a diminué le pouvoir des partis politiques en tant qu'initiateurs des débats politiques et a renforcé celle des médias. C'est dans ce contexte politique qu'est née la conception de diplomatie populaire comme principe de représentation politique, hybride d'une représentation nationale et culturelle apolitique. La conjoncture politique imposée par les règles du jeu pour les élections européennes a donc empêché les partis politiques de développer leurs conceptions collectives de l'Union européenne et a conforté le monopole discursif de l'exécutif politique sur l'Europe. Le cas des élections européennes finlandaises révèle plusieurs faiblesses du modèle Reif et Schmitt. Elles sont d'abord d'ordre structurel. Les caractéristiques des élections européennes dépendent surtout de leur position dans le champ politique national en question, pas uniquement de la position des élections dans le cycle électoral national, comme le suggèrent Reif et Schmitt ${ }^{20}$. Comme le cas finlandais le montre, elles ne signifient pas nécessairement une baisse de popularité des partis au pouvoir, ni le succès des partis protestataires ou des petits partis ${ }^{21}$. Le modèle Reif-Schmitt ne prend pas assez en compte le système électoral, qui cadre la compétition politique et détermine les caractéristiques des campagnes. La seconde faiblesse du modèle tient au fait qu'il néglige les effets qualitatifs des élections. En effet, Reif et Schmitt considèrent uniquement les effets quantitatifs : popularité des partis et taux de vote. Les élections européennes sont conçues, probablement inconsciemment, comme une extension de la politique nationale. Comme le cas finlandais le démontre, les élections permettent aussi la construction sociale de nouvelles conceptions de la représentativité politique et de la délégation politique, comme celle de la diplomatie populaire. Ces transformations qualitatives sont, à plusieurs égards, plus importantes que les changements de quelques pourcentages dans la popularité des partis politiques. Un des effets les plus visibles de l'européanisation du champ politique national finlandais est la différenciation des élections et des élites, des élections européennes et des europarlementaires. C'est par cette différenciation que le champ politique finlandais est inséré dans l'espace politique européen. Cette stratégie collective d'européanisation qu'on pourrait appeler aussi une stratégie par différenciation se distingue de celle mise en place en France, où l'européanisation du système électoral s'effectue par une extension du pouvoir des partis politiques et une simple annexion des élections européennes (intégration par extension). Ceci permet aux partis politiques français de formuler leur Europe et de l'incorporer dans leur programme politique. 


\section{NOTES}

1. Voir par exemple Jaakko Nousiainen, « EY-jäsenyyden vaikutus Suomen valtiollisten laitosten keskinäisiin toimivaltasuhteisiin » (L'effet de l'adhésion aux Communautés européennes sur les relations entre institutions étatiques finlandaises), Politiikka vol. 34, n³ , 1992, pp. 262-270 ; Eero Murto, Pekka Väänänen \& Raimo Ikonen, Sisäpiirit EUSuomessa. Unioni ja uudet eliitit. (Les milieux politiques dans l'UE-Finlande. L'Union et les nouvelles élites), Helsinki, Edita, 1996 ; Niilo Kauppi, « Kohti postabsolutista valtiota ? EU-tutkimuksen haaste politiikan tutkimukselle » (Vers un état postabsolutiste? Le défi de la recherche sur l'UE à la science politique), Politiikka, vol. 39, nº 1, 1997, pp. 60-63.

2. Murto, Väänänen, Ikonen 1996 op. cit.

3. Nousiainen, 1992, op. cit. Le processus d'adhésion à l'Union commence déjà en septembre 1991, quand le premier ministre centriste Esko Aho déclare que la Finlande commencera à se renseigner sur une éventuelle adhésion à l'Union. Cf. Jukka Heinonen, « Kansanedustajien EY-kantaan vaikuttaneet taustatekijät » (Les facteurs influençant les positions des députés sur les Communautés européennes), Politiikka, vol. $34, \mathrm{n}^{\circ} 3$, 1992, pp. 280-286.

4. Cf. Jyri Raivio dans son portrait de l'ambassadeur finlandais à Bruxelles Antti Satuli, " Ulkoasianhallinnon Stahanov » (Le Stakhanov des affaires étrangères), Helsingin sanomat, Octobre 10, 1996.

5. Sur le capital politique européen, cf. les articles d'Adrian Favell, d'Andy Smith et de Didier Georgakakis dans ce numéro.

6. Karlheinz Reif, « Reflections : European Elections as Member State Second-order Elections Revisited », European Journal of Political Research, n³1, 1997, pp. 115-124 ; Karlheinz Reif et Hermann Schmitt, « Nine Second-order National Elections - A Conceptual Framework for the Analysis of European Elections Results », European Journal of Political Research, n8, 1980, pp. 3-44.

7. Cf. Pascal Perrineau, « L'élection européenne au miroir de l'hexagone. Les résultats des élections européennes en France " pp. 229-261, in Le vote des douze. Les élections européennes de juin 1994, Paris, Presses de Science Po, 1995 ; Christian Bidégaray et Claude Emeri, «Enjeux européens et systèmes des partis politiques français », pp. 61-76, in De la Ve République à l'Europe, Paris, Presses de Science Po, 1996 ; Niilo Kauppi, «European Union Institutions and French Political Careers », Scandinavian Political Studies, $n^{\circ} 1,1996$, pp. 1-24.

8. Cf. Pia Majonen, « Kauniita ja rohkeita vai aatteellisia ammattipoliitikkoja ?(Les beaux et les courageux ou des politiques professionnels idéologues) », in Tuomo Martikainen et Kyösti Pekonen (dirs.), Eurovaalit Suomessa 1996. Vaalihumusta päätöksenteon arkeen (Les élections européennes en Finlande 1996. Du bruit électoral aux routines décisionnelles), Université de Helsinki, Département de science politique, 1999.

9. Cf. Tilastokeskus (Centre statistique de Finlande) 1999. Disponible sur http:// www.tilastokeskus.fi.

10. Pekka Pesonen, Risto Sänkiaho et Sami Borg, Vaalikansan äänivalta (La voix du peuple électeur), Helsinki, WSOY, 1993, pp. 53-69.

11. A ce sujet, cf. Majonen 1996 op. cit. 
12. Cf. Dag Anckar, « The Finnish European Election of 1996 », Electoral Studies, vol.16, $\mathrm{n}^{\circ} 2,1997$, pp. 262-265.

13. Tuomo Martikainen et Kyösti Pekonen (dirs.), Eurovaalit Suomessa 1996.

Vaalihumusta päätöksenteon arkeen (Les élections européennes en Finlande 1996. Du bruit électoral aux routines décisionnelles), Université de Helsinki, Département de science politique, 1999.

14. Tilastokeskus 1999 op. cit.

15. Jean-Dominique Merchet, « Trois questions à Ari Vatanen », Libération, 8 juin 1999, p. 15.

16. Erkki Pennanen, « Herätys, vaalit ovat ovella » (Réveillez-vous, les élections sont à la porte), Helsingin sanomat, 1er Juin 1999, p. 2.

17. Eija-Riitta Korhola, « Asiat kiinnostavat politiikkaa enemmän » (Le devoir m’intéresse plus que la politique), Helsingin sanomat, 15 juin 1999, p. B3.

18. Tarja Ahokas, « Kiva juttu » (Chouette !), Iltasanomat, 14 juin 1999, p. B6.

19. Hokkanen, Kari. « Hyvä mies » (Un honnête homme). Ilkka, 17.10.1996.

20. Pour le cas de la France, cf. Niilo Kauppi, « Globalization, Social Movements, and the Construction of Europe : The Example of the European Parliament Elections in France ", Center for European Studies Working Papers \# 74, Harvard University, août 2000, 51 p. Disponible sur http://www.columbia.edu/cu/cup/ciaopromo/index.html.

21. Cf. aussi Elina Härkönen, « Ensimmäiset europarlamenttivaalit Suomessa : toisen asteen vaalit?» (Les premières élections européennes en Finlande : des élections de second ordre ?) in Martikainen \& Pekonen (dirs.) op. cit.

INDEX

Mots-clés : construction européenne, démocratie

Index géographique : Europe du Nord

Index chronologique : 1999 